\title{
Contraceptive needs of female sex workers in Kenya - A cross-sectional study
}

Elizabeth G. Sutherland ${ }_{\S}^{\star}$, Jane Alaii ${ }^{\dagger}$, Sharon Tsui ${ }^{\ddagger}$, Stanley Luchters ${ }^{\S}, \#$, Jerry Okal ${ }^{\#}$, Nzioke King'ola ${ }^{\#}$, Marleen Temmerman ${ }^{\S}$ and Barbara Janowitz

*MEASURE Evaluation Project, Carolina Population Center, University of North Carolina at Chapel Hill, Chapel Hill, NC, USA; ${ }^{\dagger}$ Family Health International, Nairobi, Kenya; ${ }^{\star}$ Family Health International, Research Triangle Park, NC, USA; ${ }^{\S}$ University of Ghent, Ghent, Belgium; and ${ }^{\#}$ International Centre for Reproductive Health, Mombasa, Kenya

A B S T R A C T Background and objectives Female sex workers (FSWs) are thought to be at heightened risk for unintended pregnancy, although sexual and reproductive health interventions reaching these populations are typically focused on the increased risk of sexually transmitted infections. The objective of this study of FSWs in Kenya is to document patterns of contraceptive use and unmet need for contraception.

Methods This research surveys a large sample of female sex workers $(N=597)$ and also uses qualitative data from focus group discussions.

Results The reported level of modern contraceptives in our setting was very high. However, like in other studies, we found a great reliance on male condoms, coupled with inconsistent use at last sex, which resulted in a higher potential for unmet need for contraception than the elevated levels of modern contraceptives might suggest. Dual method use was also frequently encountered in this population and the benefits of this practice were clearly outlined by focus group participants.

Conclusion These findings suggest that the promotion of dual methods among this population could help meet the broader reproductive health needs of FSWs. Furthermore, this research underscores the necessity of considering consistency of condom use when estimating the unmet or undermet contraceptive needs of this population.

K E Y W O R D S Contraception; Prostitution; Female; Reproductive history; Kenya

I N T R O D U C T I O N

Female sex workers (FSWs) are often targeted by public health interventions designed to prevent sexually transmitted infections (STIs), including infec- tion with the human immunodeficiency virus (HIV). However, such targeted programmes sometimes overlook the broader reproductive health needs of these

Correspondence: Elizabeth Sutherland, MEASURE Evaluation Project, Carolina Population Center, University of North Carolina at Chapel Hill, 206 W. Franklin St. Chapel Hill, NC, USA. Tel: +1 919843 3772. E-mail: beth_sutherland@unc.edu 
women. As a result, inadequate attention has been paid to the issue of unintended pregnancy, another heightened reproductive health risk among sex workers ${ }^{2}$.

Few studies have reported on pregnancy desires and contraceptive use among FSWs. The limited amount of existing research has shown high incidences of unintended pregnancy and a low contraceptive prevalence in this particular group ${ }^{3-6}$. All studies report that sex workers who do use contraception resort most often to condoms yet the latter are not consistently used in all sex acts ${ }^{3,5-9}$. FSWs may have difficulties in negotiating the consistent use of condoms with clients, and also may deliberately use condoms with some types of partners (such as casual clients) while deliberately not using condoms with other types of partners (such as emotional partners or regular clients) ${ }^{10}$.

The consistency of condom use is a critical consideration when evaluating its role in reducing unmet need for contraception, not to mention HIV/ STI prevention. With 'typical' use, condoms have only a 79-85\% effectiveness rate at preventing pregnancy, which is lower than the typical use effectiveness of many other modern methods ${ }^{11}$. As commonly measured and reported, unmet need for contraception does not take into account the correct and consistent use of a method, only whether a method use is reported. However, accurately documenting and addressing FSWs' contraceptive needs is essential given their many and varied reproductive health risks, including unintended pregnancy and mother-to-child HIV transmission.

We investigated patterns of recent self-reported contraceptive use and unmet contraceptive needs among FSWs in two urban areas of Kenya. This study also assesses the impact of inconsistent condom use on the measurement of unmet need for contraception and describes the experience with dual method use (the use of multiple contraceptive methods simultaneously) among FSWs who are served by active condom promotion programmes.

\section{METHODS}

The research took place from June to December 2008, within the Naivasha and Changamwe districts of Kenya's Rift Valley and Coast Provinces, respectively. These urban to semi-urban districts are known for having a concentrated FSW population, partly due to the port and tourist trade in Changamwe, and to truck drivers and seasonal workers in flower farms in Naivasha. FSW communities in both districts are being actively served by long-running and well established HIV and STI prevention programmes. These programmes are administered by two different organisations but are similar in nature, having an emphasis on condom promotion and peer education. The programme in Changamwe is administered by the International Centre for Reproductive Health, Kenya (ICRH-K) and that in Naivasha by Lifebloom Services International (LBSI). In both interventions, peer educators actively promote HIV testing and counselling, and condom use.

We utilised a mixed-method cross-sectional study design consisting of survey interviews and semistructured focus group discussions (FGDs) with FSWs aged 16-45 years. A FSW is defined as any sexually active woman who reported receiving money or goods in exchange for sex within the last six months as a part of her livelihood. Beside sociodemographic information, the individual interviews assessed FSWs' fertility desires, unmet need for contraception, and knowledge, attitudes, and experiences relating to the use of family planning (FP) methods in the past 30 days. Focus group discussions were conducted to explore normative perceptions and behaviours/practices surrounding FP as well as to investigate sex workers' outlook on FP methods and services.

A targeted snowball sample of 597 individuals were interviewed across the two research sites $(n=300$ in Changamwe and $n=297$ in Naivasha). The FGDs involved four groups, each consisting of 8-12 participants, resulting in a total of eight discussions and eight transcripts. Written informed consent was privately obtained from both interviewees and focus group participants.

Peer educators spread the word that a team of interviewers could be found at a secure site and that they were keen on speaking with FSWs. Interested women approached the interview team. Those who met the study inclusion criteria (FSW aged 16-45 years) were invited to participate at random in either the survey or a FGD, with every other eligible participant asked to take part in a FGD until the FGD discussions were full or complete. Participation in one type of interview precluded participation in the other. 
All interviews and FGDs took place at a neutral, confidential location secured by the research team in the study sites. All interviews were conducted in the national language, Swahili, by trained interviewers. Similarly, a trained duo of a focus group moderator and a note taker conducted all FGDs. The same focus group research pair conducted all focus groups in both study sites to enhance consistency, and FGDs were not held concurrently in space and time with individual interviews.

Cash compensation at a standard flat rate of Ksh 300 (approximately USD $\$ 4.29 / € 2.90$ at the time of data collection) was provided to all study participants upon arrival at the interview location and in exchange for their participation voucher. This compensation was provided to compensate for participants' transportation costs to the interview site, and was approved by two institutional review boards, including the Family Health International's Protection of Human Rights Committee and the Kenyatta National Hospital Ethical Review Committee.

At the close of the interview or focus group, all participants received information on readily available service delivery points for FP and HIV Counselling and Testing services.

\section{Data management and analysis}

All interview data were double entered into EpiData v. 3.1 (EpiData Association) by trained data entry specialists. Data were then cleaned and queried using Stata v. 9.0 (StataCorps). Descriptive data analyses were conducted and were verified by an independent analyst before being considered final. Data analysis is restricted to descriptive statistics only, and no probability-based inferential statistics (such as comparison testing or regression analysis) were calculated.

Survey respondents were considered to have an immediate unmet need for contraception if they were not currently pregnant, not currently using a modern FP method, and did not desire a pregnancy within the next two years. As the survey did not assess the exclusivity and duration of breastfeeding, breastfeeding status was not included as a component of unmet need. In the revised estimate of unmet need, we also included women who were currently relying on condoms alone but used them inconsistently. Inconsistent condom-only users were those who were using only the male or female condom but reported not using a condom at last sex with either a client or an emotional (non-paying) partner. While the Demographic and Health Surveys (DHS) currently consider pregnant women as having an unmet need for contraception if the pregnancy was unplanned ${ }^{12}$, for the purposes of this analysis we restricted the analysis to those women with an immediate need for contraception, excluding pregnant women who are not in a position to immediately adopt a contraceptive method.

FGDs were digitally recorded, uploaded to a laptop computer, transcribed verbatim, and translated from Swahili to English by the moderator and note taker. Transcriptions and translations were reviewed for quality by the interview team. The analysis team performed qualitative analyses with NVivo v. 7.0 (QSR International Pty Ltd) qualitative data analysis software. A content-driven theme approach was used for analytic review of the FGD data. Transcripts were read and re-read to identify recurrent themes and to develop a coding tree. Once all the transcripts were coded, memos and display matrices were developed to examine each code in detail for sub-themes, nuances, and patterns across the interviews.

\section{RES U L T S}

\section{Demographic characteristics and reproductive history}

The average survey respondent was in her late 20s, with at least one living child, and did not currently live with a partner with whom she was emotionally involved, although about half the women reported having such a partner (Table 1). Less detailed background information was collected from FGD participants, although the latter did not significantly differ from survey respondents by age or parity (data not shown), and were recruited at the same time and in the same manner as those.

Nine percent of FSWs $(N=597)$ stated they were infected with HIV. The average age of sex work initiation in both sites was 20 years and a minority of FSWs reported having a source of income other than sex work. The overwhelming majority of sex workers in both Naivasha and Changamwe stated they primarily relied on bars (78\% in Naivasha and 64\% in Changamwe) as an 'easy place to meet clients'. However, 9\% of sex workers in Changamwe reported 
Table 1 Demographic and sex work characteristics of survey interview participants

\begin{tabular}{|c|c|c|c|c|c|c|}
\hline & \multicolumn{6}{|c|}{ Mean (SD) or \% } \\
\hline & \multicolumn{2}{|c|}{$\begin{array}{c}\text { Overall } \\
(N=597)\end{array}$} & \multicolumn{2}{|c|}{$\begin{array}{l}\text { Naivasha } \\
(n=297)\end{array}$} & \multicolumn{2}{|c|}{$\begin{array}{l}\text { Changamwe } \\
(n=300)\end{array}$} \\
\hline & Mean or $\%$ & $S D$ & Mean or \% & $S D$ & Mean or \% & $S D$ \\
\hline \multicolumn{7}{|l|}{ Demographic characteristics } \\
\hline Age & 27.4 & 6.9 & 27.3 & 6.6 & 27.6 & 7.3 \\
\hline Years of education & 8.7 & 3.0 & 8.5 & 2.5 & 8.8 & 3.4 \\
\hline Has living children & 82.9 & - & 80.5 & - & 85.3 & - \\
\hline Number of living children & 1.8 & 1.3 & 1.9 & 1.4 & 1.8 & 1.3 \\
\hline Has a current emotional partner & 53 & - & 62 & - & 44 & - \\
\hline $\begin{array}{l}\text { Currently lives with a partner with } \\
\text { whom she is emotionally involved }\end{array}$ & 4 & - & 5 & - & 2 & - \\
\hline HIV-positive (self-disclosed) & 9 & - & 8 & - & 11 & - \\
\hline \multicolumn{7}{|l|}{ Sex work characteristics } \\
\hline Age of sex work initiation & 20.3 & 5.2 & 20.0 & 4.0 & 20.7 & 6.0 \\
\hline Has income other than sex work & 34 & - & 46 & - & 22 & - \\
\hline $\begin{array}{l}\text { Average income from sex work in } \\
\text { the last } 7 \text { days (in } \mathrm{KSH})^{*}\end{array}$ & 2244 & 4817 & 1351 & 889 & 3124 & 6620 \\
\hline Number of clients in last 7 days & 7.3 & 5.7 & 5.3 & 3.5 & 9.3 & 6.7 \\
\hline Recruits clients mostly in bars/discos & 71 & - & 78 & - & 64 & - \\
\hline Recruits clients mostly in brothels & 7 & - & 4 & - & 9 & - \\
\hline Recruits clients mostly in brew dens & 5 & - & 0 & - & 9 & - \\
\hline
\end{tabular}

*70 KSH $=1 \mathrm{USD}=0.68 €$ at the time of data collection.

SD: standard deviation

being primarily brothel-based, and another 9\% primarily meeting their clients in illegal brew dens. In Naivasha, only $4 \%$ of respondents mentioned being brothel based, and none allegedly met clients in brew dens. The remaining sex workers in both sites reported a combination of primary client recruitment locations including the street (5\%), home $(5 \%)$, and other places (including the beach, petrol stations, workplaces, etc.). Changamwe sex workers recalled both a greater number of clients (9.3 vs. 5.3) and a higher weekly income (3124 KSH vs. $1351 \mathrm{KSH}$ per week) from sex work than their counterparts in Naivasha, though the standard deviations are large. The range of the number of partners reported in Naivasha was zero to 21 , with a median of five, and in Changamwe was zero to 47 , with a median value of eight.

FSWs from both study sites frequently recounted histories including unintended pregnancies and induced abortion (Table 2). Almost half of all respondents described having had at least one unintended pregnancy in the past, and more than a third of respondents told of having had an induced abortion (an illegal and often unsafe procedure) at some point in the past.

Qualitative data further highlight the realities associated with unintended pregnancy among FSWs. Informants reported that unintended pregnancies were a common occurrence among FSWs, with distressing economic and personal consequences, including loss of work and clients, potential for domestic violence from a boyfriend or partner displeased with the pregnancy, and the burden posed by the financial expense of raising another child. As one FGD participant described:

it is important for her [a sex worker] to prevent pregnancy because her life as it is right now is stressful. She does not have anyone to depend on except herself, there is no one to help with taking care of her children, she has to lock her house at night when she is out there soliciting and if she is doing it not far away, she will have 
Table 2 Past experience with unintended pregnancy and induced abortion, and current fertility desires of survey interview participants, by study site (\%)

\begin{tabular}{|c|c|c|c|}
\hline & $\begin{array}{c}\text { Overall } \\
(N=597)\end{array}$ & $\begin{array}{l}\text { Naivasha } \\
(n=297)\end{array}$ & $\begin{array}{c}\text { Changamwe } \\
(n=300)\end{array}$ \\
\hline \multicolumn{4}{|l|}{ Reproductive history } \\
\hline Ever been pregnant & 88 & 85 & 92 \\
\hline Ever had an unintended pregnancy & 52 & 46 & 59 \\
\hline Ever had an induced abortion & 37 & 32 & 43 \\
\hline \multicolumn{4}{|l|}{ Current fertility desires } \\
\hline Not pregnant, wants no more children ever & 60 & 54 & 64 \\
\hline Not pregnant, wants no more children within two years & 16 & 24 & 9 \\
\hline Not pregnant, wants more children within two years & 20 & 15 & 25 \\
\hline Currently pregnant & 4 & 7 & 2 \\
\hline
\end{tabular}

to keep on running back and forth just to check how her children are doing. It will be difficult if she [gets] pregnant.

Also, informants openly discussed the practice of induced abortion as an option to be considered when faced with an unintended pregnancy. For example, one FGD informant said, when asked what a FSW would do when faced with an unintended pregnancy, that:

what will happen is that [she] will just remove the pregnancy. She will just remove it. She already has another child and now she is pregnant, landlord comes calling, this child is crying of hunger and maybe she has the kind of pregnancy which causes a lot of stress... it is better to remove it and continue with life.

Other FSWs reported being pressured to procure an abortion by partners as this FGD respondent described, ' ... another [partner] will give you money and tell you to remove it and, if not, will harass you until you comply'. Many FSWs described weighing the difficulties of an unintended pregnancy against possible abortion complications as another FGD respondent explained, '... you have seen the need to keep the baby and not have an abortion which may be risky not just to your health but your life too'.

\section{Contraceptive use}

Overall, 16\% of survey participants reported wishing to wait at least two years before having more children, and $60 \%$ of the women expressed a desire to have no pregnancies at any time in the future (Table 2). Furthermore, the current use of modern contraceptives was common (93\%), with 54\% reporting the use of a modern method other than condoms, usually injectables or pills (Table 3). While 39\% of all respondents recounted using a male or female condom by itself, $38 \%$ of the total sample described using a male condom together with another modern method. This second method was usually an injectable but could also be the pill.

Respondents recognised the dual protection benefits of the condom for disease and pregnancy prevention. As a focus group informant explained:

I also think [a FSW] should ... use a condom. Here she will have two things; one she will have protection against infection from her clients, and two do family planning. She must use a condom to protect herself from infections and at the same time not add more children to the ones she already has.

Other respondents were able to clearly articulate the benefits of dual method use, rather than relying on condoms alone. For example, as this focus group respondent said, 'she [a FSW] will use condoms mostly to prevent STIs, but she is already using another method to prevent pregnancy'. This appreciation among sex workers for the benefits of dual protection via condom use and dual method use is reflected in the high rates of condom use and dual method use observed in this population. 
Table 3 Contraceptive practices among survey respondents, by study site (\%)

\begin{tabular}{|c|c|c|c|}
\hline & Overall $N=597$ & Naivasha $N=297$ & Changamwe $N=300$ \\
\hline \multicolumn{4}{|l|}{ Reported current contraceptive use } \\
\hline No method & 7 & 6 & 10 \\
\hline Condoms and another modern method & 38 & 34 & 41 \\
\hline Condoms only & 39 & 34 & 44 \\
\hline Modern method other than condoms, only & 16 & 26 & 5 \\
\hline \multicolumn{4}{|l|}{ Method mix* } \\
\hline Male condom & 75 & 68 & 83 \\
\hline Female condom & 6 & $<1 \%$ & 12 \\
\hline Injectables & 39 & 46 & 32 \\
\hline Pills & 11 & 10 & 11 \\
\hline Implants & 2 & 2 & $<1 \%$ \\
\hline Other modern method & 2 & 2 & 3 \\
\hline \multicolumn{4}{|l|}{ Condom use consistency } \\
\hline $\begin{array}{l}\text { Used a condom at last sex with a partner } \\
\text { with whom she was emotionally involved }\end{array}$ & 41 & 40 & 42 \\
\hline Used a condom at last sex with a paying client & 90 & 98 & 82 \\
\hline $\begin{array}{l}\text { Negotiating condom use with clients is } \\
\text { 'difficult' or 'impossible' }\end{array}$ & 46 & 39 & 52 \\
\hline
\end{tabular}

* Method mix sums to greater than $100 \%$ because respondents could report more than one method.

Many focus group participants and survey respondents report that they used or would like to use methods that were effective but did not require daily intervention, such as injectable contraception or an implant. It was often mentioned that sex work circumstances interfere with the correct and consistent use of other methods. Focus group informants described getting drunk or staying out with a partner or client for several days which prevented or would prevent them from adhering to the daily regimens of the pill, and/or correctly and consistently using the condom. For example, an informant discussed difficulty adhering to the pill when:

You go out there to drink alcohol and end up drunk. You will oversleep and cannot even remember to go home. If that happens, then it is not always possible to remember taking the pill which you have to swallow every day.

And another informant explained, 'Let's say you are supposed to take it every evening or morning, you get a client who keeps you all week long, you did not take them with you, you simply forget'. Another FGD respondent expressed the benefit of injectable contra- ception over a condom for sex workers who cannot negotiate condom use, saying, 'Yes, she is ready to use [injectable contraception] because maybe a client would not want to use a condom during sex... At the time, [a FSW] would have an assurance that at least the injection received earlier on has saved the day'.

As described above, many FGD participants mentioned that condoms and pills could be easily forgotten. Many respondents also noted that condoms could tear during intercourse. For example, one respondent described the situation of a FSW who found herself pregnant 'because she has very many and different clients, maybe she has been with this one client today and the condom burst, the previous day she may have not used a condom at all'. However, both these methods were frequently mentioned by FGD respondents as 'the most convenient for sex workers' because they are readily available. As one FGD respondent explained about forgetting her pack of pills, '...we are in town and there are many chemists around, all I'll do is rush to one and buy a new pack'. In addition to convenience, condoms, as discussed above, were valued for their dual protection attributes. Also, the ability to take pills as emergency contraception (EC) or to buy pre-packaged EC 
pills was also highlighted as a benefit by many respondents.

Few FGD informants would like to use the intrauterine device (IUD) because many mistakenly perceived it as being 'dangerous' for sex workers. It was commonly believed that the IUD could be displaced through rough sex and cause problems as is highlighted by the following excerpt from a conversation between three focus group respondents; R8: '[One contraceptive method available is the] coil [IUD], but you could use it with someone who is rough and hurts you'. R6: 'Or his penis is big and displaces the coil inside you'. R7: 'Maybe the man is drunk or high on bhang and he pushes the coil so hard inside her that he disfigures it'. Also, a few informants were concerned that clients could feel the strings of the IUD and react negatively. For example, one respondent said 'she [a sex worker] does not want to use the coil because who [a client] may feel it during sex and wonder what it could be'.

\section{Unmet need for contraception and condom use consistency}

Unmet need for contraception, was very low (3.8\%) among survey respondents. A closer analysis reveals that this extremely low level of unmet need arises from the high levels of self-reported contraceptive use, in particular the use of male condoms. However, the consistency of condom use in this population is called into question given the relatively low reported rates of condom use at last sex with emotional partners (41\%) as opposed to clients (90\%) (Table 3) and the sizeable proportion of survey respondents (46\%) who reported condom use negotiation with clients to be 'difficult' or 'impossible'.

Qualitative data supported survey findings on inconsistency of condom use. FGD informants detailed many difficulties sex workers faced in negotiating condom use and/or using condoms correctly and consistently. Difficulties described included FSWs foregoing condom use with clients for higher payment of sex, fear of sexual violence or force, forgetting to use condoms because of substance use, and not being able to negotiate condom use with emotional partners for issues related to trust. For example, one respondent explained, 'one may get a client or clients but they do not want to use protection [a condom], and they force you'.
The calculation of unmet need was revised (as described in the methods section above) to include those women who were not currently pregnant, who did not wish to have a child in the next two years, who reported using condoms as their only contraceptive method in the last 30 days, and who also stated they had not used a condom at last sex with either a partner with whom they were emotionally involved or a client. This recalculation gives a potential unmet need of $13.6 \%$ among the FSWs surveyed almost four times the unmet need when not accounting for condom use consistency.

\section{I S C U S S I O N}

This study contributes to the literature both conducting a primary survey of a large sample of FSWs and by supporting and nuancing quantitative results with the qualitative findings from focus group discussions. This work builds on earlier, mostly secondary, analyses of the contraceptive needs of FSWs ${ }^{4-6}$. The only other mixed-method, primary study of the unmet needs of FSWs of which the authors are aware was conducted in Southeast $\mathrm{Asia}^{3}$. Additionally, working in study areas with active STI prevention and peer-educator interventions provided this study with two additional advantages. First, the ongoing STI prevention efforts of ICRH-K and LBSI have built trust and credibility in the FSW community which led to closer participation and faster recruitment. Second, our estimate of unmet need for contraception in this population demonstrates the degree of need which remains despite heavy condom promotion and active HIV/STI prevention messaging.

The many sexual partners on a weekly basis reported by survey respondents in this study highlight the vulnerability of FSWs to both unintended pregnancy and STIs, including HIV. In our sample of FSWs, most respondents had children already, and there was a high desire to delay and limit births. Furthermore, our study population practised contraception on a wide scale.

Unlike previous studies which showed high unmet needs for contraception in sex worker populations, the estimated unmet need for contraception in the FSWs we sampled, based on any reported use of a modern method, was low $(<5 \%)$. A revised calculation of unmet need which includes inconsistent (no condom use at last sex) condom users resulted in a greater 
(almost quadrupled) level of unmet need in this population. This revised estimate may not be entirely exact and hence may neither precisely reflect actual behaviour nor correct for potential response biases (a difficulty inherent in sexual behaviour research ${ }^{13-15}$ ). Yet, it does reflect the importance of considering the correct and consistent use of condoms when describing the contraceptive needs of this population. In fact, this revised estimate of unmet need may still underestimate the actual unmet need if the FSWs surveyed overstated condom use in the last 30 days, and/or overstated condom use at last sex, or if condom use at last sex is not 'typical' for the population sampled. Overstatement of condom use at last sex seems particularly likely in this study, given the apparent conflict that exists between the women's reports of nearly universal condom usage with clients, and their frequent description of condom use with clients as being 'difficult' or 'impossible'. The reported high levels of condom use are perhaps unsurprising in our two study sites, which are actively targeted by current and ongoing STI prevention programmes. The study area FSWs may indeed show higher than average levels of condom use compared to similar women in other parts of Sub-Saharan Africa or their heightened exposure to condom messages may promote a social desirability bias.

To prevent unintended pregnancies in this group, there remains a great need for promoting alternative and potentially more effective contraceptive methods, particularly non-daily use or non-coitally dependent methods such as injectables and implants, in addition to condoms. The promotion of dual method use would lessen the risk of unintended pregnancy in the many situations when FSWs cannot correctly and consistently use condoms at every sex act or take pills every day. Most respondents in our study wished to limit childbearing by avoiding any future pregnancy. However, contraceptives used consisted mainly of condoms and pills. Although focus group participants were able to articulate some advantages of condoms and pills, the desirability of longer acting methods, especially combined with condoms as part of a dual method strategy to prevent both pregnancy and disease, was often mentioned, as well. This suggests there may be some 'undermet' need for long-acting and permanent methods, and dual method use, among this group.

Promoting dual method use to a population at high risk of unintended pregnancy and STIs (including
HIV) may raise fears that, once using another contraceptive method, FSWs will be less inclined to use also a condom ${ }^{16}$. However, in this study, focus group participants repeatedly mentioned the advantages of dual protection over condoms only. Survey results also indicated a sizeable proportion of women who reported dual method use in the last 30 days. Further, the survey indicated that some women who desired a pregnancy were still using condoms and many women were actively separating their use of condoms for HIV/STI prevention and for pregnancy prevention. It seems likely this separation could be resulting from the successful promotion of condoms for disease prevention by active STI prevention activities ongoing in the study area. This formative information suggests that a dual methods message could be appreciated and well-received by the FSWs whom we interviewed.

The finding that many FSWs are using contraception but remain in need due to incorrect and inconsistent use of contraceptives (especially condoms but also pills) highlights the need to define a more accurate way of measuring unmet need for contraception in this population. In this study unmet need was first measured in the usual way, excluding women who reported having used a modern contraceptive in the last 30 days. However, considering contraceptive users as having a 'met contraceptive need' becomes unrealistic when considering FSWs who may be using condoms or other methods with certain partners but not others, who have a large number of partners, and who may be experiencing difficulties in using their method correctly and consistently. This complexity underscores the reality that the reproductive health needs of FSWs are diverse and interconnected, and that efforts to address STIs, HIV, and unintended pregnancy should be made within an integrated reproductive health framework.

Broader interpretations of the findings of this study must be made with caution. Given that sex work is illegal in Kenya and highly stigmatised, we were unable to randomly sample this hard-to-reach population. Therefore, inferences can only be drawn for study participants and cannot be generalised to the larger population of FSWs in Kenya. Further, like all sexual behaviour surveys this study is subject to response biases $^{13,15}$, including a potential underestimate of the proportion of the participants who were HIV positive, a possible overestimate of condom use 
and condom use at last sex, and a probable underestimate of unintended pregnancies and induced abortions experienced in the past. In fact, the incidence of HIV reported in this study is markedly lower than that in studies among FSWs in Kenya recently carried out by Luchters et al. ${ }^{1}$ and Vandenhoudt et al. (unpublished, 2010), while rates of condom use are similar to those found in other studies of Kenyan FSWs (Vandenhoudt et al. unpublished, 2010). It is also possible that women lied about exchanging money for sex in order to obtain the compensation offered. However, efforts to limit this was made by asking for referrals from peer educators, using open-ended screening questions to determine study eligibility, the self-identification (and selfpresentation at a pre-selected interview site with no guarantee of eligibility), and the selection of a compensation amount that was in line with similar studies. Even with these limitations, however, our findings of high contraceptive use, low condom use consistency, and high effective levels of unmet need for contraception which could be ameliorated by dual method use have implications for the likely contra- ceptive needs of this stigmatised and vulnerable population.

\section{A C K N O W L E D G E M E N T S}

This work was completed with support from the United States Agency for International Development (USAID) to Family Health International (FHI) under cooperative agreement \#GPO-A-00-05-00022-00 and while the first (and corresponding) author was affiliated with FHI. The views expressed in this paper, however, are solely those of the named authors. We gratefully acknowledge the assistance of our colleagues who were instrumental to the fielding of this study, including Mackenzie Green, Jennifer Liku, Dominick Shattuck, Katie Tweedy, and Saade Abdallah, as well as our team of research assistants. We also greatly appreciate the technical review provided by Theresa Hatzell Hoke.

Declaration of interest: The authors report no conflicts of interest. The authors alone are responsible for the content and writing of the paper.
1. Luchters S, Chersich MF, Rinyiru A, et al. Impact of five years of peer-mediated interventions on sexual behavior and sexually transmitted infections among female sex workers in Mombasa, Kenya. BMC Public Health 2008;8:143 doi:10.1186/14712458-8-143.

2. Rekart M. Sex-work harm reduction. Lancet 2005;366: 2123-34.

3. Delvaux T, Crabbé F, Seng S, Laga M. The need for family planning and safe abortion services among sex workers seeking STI care in Cambodia. Reprod Health Matters 2003;11:88-95.

4. Elmore-Meegan M, Conroy RM, Agala CB. Sex workers in Kenya, number of clients and associated risks: An exploratory survey. Reprod Health Matters 2004;12: 50-7.

5. Feldblum PJ, Nasution MD, Hoke TH, et al. Pregnancy among sex workers participating in a condom intervention trial highlights the need for dual protection. Contraception 2007;76:10510.
6. Khan MR, Turner AN, Pettifor A, et al. Unmet need for contraception among sex workers in Madagascar. Contraception 2009;79:221-7.

7. Kayembe PK, Mapatano MA, Busangu AF, et al. Determinants of consistent condom use among female commercial sex workers in the Democratic Republic of Congo: Implications for interventions. Sex Transm Infect 2008;84:202-6.

8. Murray L, Moreno L, Rosario S, et al. The role of relationship intimacy in consistent condom use among female sex workers and their regular paying partners in the Dominican Republic. AIDS Behav 2007;11:463-70.

9. Thomsen SC, Ombidi W, Toroitich-Ruto C, et al. A prospective study assessing the effects of introducing the female condom in a sex worker population in Mombasa. Sex Transm Infect 2006;82:397-402.

10. Ngugi EN, Chakkalackal M, Sharma A, et al., Kibera HIV Study Group. Sustained changes in sexual behavior by female sex workers after completion of a randomized HIV prevention trial. J Acquired Immune Defic Syndr 2007;45:588-94. 
11. Hatcher RA, Trussell J, Stewart F, et al., eds. Contraceptive technology, 18th edn. New York: Ardent Media, Inc. 2004.

12. Rutstein SO, Rojas G. Online guide to DHS statistics. 2006. Retrieved on 4 January 2010 from: http:// www.measuredhs.com/help/Datasets/index.htm.

13. Catania JA, Turner H, Pierce RC, et al. Response bias in surveys of AIDS-related sexual behavior. In: Ostrow DG, Kessler RC, eds. Methodological issues in AIDS behavioral research. New York: Plenum Press 1993.

14. Gallo MF, Behets FM, Steiner MJ, et al. Validity of selfreported 'safe sex' among female sex workers in
Mombasa, Kenya - PSA analysis. Int J STD AIDS 2007;18:33-8.

15. Gibson DR, Hudes ES, Donovan D. Estimating and correcting for response bias in self-reported HIV risk behavior. J Sex Res 1999;36:96-101.

16. Haddock S, Hardee K, Gay J, et al. Comprehensive HIV prevention: Condoms and contraceptives count. Pop Action Int. 2008. Retrieved 13 October 2010 from: http://www.populationaction.org/Publications/ Reports/Comprehensive_Hiv_prevention/Summary. shtml. 